\title{
Double Bracket Equations and Geodesic Flows on Symmetric Spaces
}

\author{
Anthony M. Bloch ${ }^{1, \star}$, Roger W. Brockett ${ }^{2, \star \star}$, Peter E. Crouch ${ }^{3, \star \star \star}$ \\ 1 Department of Mathematics, University of Michigan, Ann Arbor MI 48109, USA \\ 2 Division of Applied Sciences, Harvard University, Cambridge, MA 02138, USA \\ 3 Center for Systems Science and Engineering, Arizona State University, Tempe, AZ 85287, USA
}

Received: 23 July 1996/ Accepted: 16 December 1996

\begin{abstract}
In this paper we consider the geometry of Hamiltonian flows on the cotangent bundle of coadjoint orbits of compact Lie groups and on symmetric spaces. A key idea here is the use of the normal metric to define the kinetic energy. This leads to Hamiltonian flows of the double bracket type. We analyze the integrability of geodesic flows according to the method of Thimm. We obtain via the double bracket formalism a quite explicit form of the relevant commuting flows and a correspondingly transparent proof of involutivity. We demonstrate for example integrability of the geodesic flow on the real and complex Grassmannians. We also consider right invariant systems and the generalized rigid body equations in this setting.
\end{abstract}

\section{Introduction}

In this paper we consider the geometry of Hamiltonian flows, and, in particular, geodesic flows, on the cotangent bundle of coadjoint orbits of compact Lie groups and on symmetric spaces. The key idea here is to study Hamiltonians formed from the normal metric on orbits of the group action. This yields Hamiltonian flows in a symmetrical coupled double (or double double) bracket form (the bracket being the Lie algebra bracket). In contrast to the work of Bloch, Flaschka and Ratiu [1990] and Bloch, Brockett and Ratiu [1992] for example, where the double bracket flow is a gradient flow and coincides in certain cases with the restriction of a Hamiltonian flow to a submanifold, here the equations are Hamiltonian with no restriction involved. This double bracket form

\footnotetext{
* Research partially supported by National Science Foundation PYI grant DMS-91-57556, AFOSR grants F49620-93-1-0037 and F49620-96-1-0100 and a Guggenheim Fellowship

$\star \star$ Work supported in part by the NSF under Engineering Research Center Program NSF D CRD-8803012, USARO grant DAAL03-92-G-0164, DARPA, AFOSR grant F49620-92-J-0466 and ONR grant N00014-92$\mathrm{J}-1887$

$\star \star \star$ Work supported in part by NSF grant DMS 91011964 and NATO grant CRG 910926
} 
turns out to be particularly useful for analyzing integrabilty according to the method of Thimm [1983] and as formalized by Guillemin and Sternberg [1983, 1984]. One gets a quite explicit form for the relevant commuting flows and a particularly transparent proof of involutivity. We demonstrate for example integrability of the geodesic flow on the real and complex Grassmannians. Extending the analysis to right invariant metrics, we also show the full (unreduced) generalized rigid body equations on $S O(n)$ may also be written in a dynamically symmetric form. Further, we show that this gives a method of deducing the canonical symplectic form on $T^{*} S O(n)$ from the symplectic form on the cotangent bundle of a coadjoint orbit. We remark also that this formalism turns out to be useful in optimal control problems and indeed these ideas were originally inspired by the work in Brockett [1994]. We discuss the optimal control aspects in related work, Bloch and Crouch [1995], and in forthcoming work on higher order variational problems (with Leite).

The structure of the paper is as follows: in Sect. 2 we consider the symplectic structure on the cotangent bundles of coadjoint orbits and derive the geodesic flow equations with kinetic energy given by the normal metric, in Sect. 3 we extend the analysis to Grassmannians, in Sect. 4 we consider the generalized rigid body problem, and in Sect. 5 we consider integrability according to the method of Thimm.

\section{Geodesic Flows}

In this section we shall derive an explicit formula for the geodesic flow on an orbit of a compact Lie group. We begin however by considering the flow with respect to a particular Hamiltonian on a linear space, the cotangent bundle of the corresponding compact Lie algebra.

Let $\mathfrak{g}$ be a complex semisimple Lie algebra, $\mathfrak{g}_{\mathfrak{u}}$ its compact real form, and $G_{u}$ the corresponding compact group. Let $\mathfrak{g}_{\mathfrak{n}}$ be its normal real form.

Let $\|\cdot\|=<\cdot, \cdot>^{1 / 2}$ be the norm induced on $\mathfrak{g}_{\mathfrak{u}}$ by the negative of the Killing form $\kappa(\cdot, \cdot)$ on $\mathfrak{g}$ and let $\mathrm{V}$ be a smooth function on $\mathfrak{g}_{\mathfrak{u}}$.

We have

Theorem 2.1. Let $\omega$ be the standard symplectic structure on $T^{*} \mathfrak{g}_{\mathfrak{u}}$. Consider the Hamiltonian

$$
H(x, p)=1 / 2\|[p, x]\|^{2}+V(x),
$$

where $V(x)$ is any smooth function on $\mathfrak{g}$ and $p$ is a momentum variable viewed as lying in $\mathfrak{g}$ by indentifying $\mathfrak{g}$ with its dual. The Hamiltonian equations of motion are

$$
\begin{aligned}
& \dot{x}=[x,[p, x]]=-[x,[x, p]], \\
& \dot{p}=[p,[p, x]]-\frac{\partial V}{\partial x} .
\end{aligned}
$$

Proof. Let $\xi=(\delta x, \delta p)$ denote an arbitrary tangent vector to $T^{*} \mathfrak{g}_{\mathfrak{u}}$ and denote the Hamiltonian vector field corresponding to $H$ by $X_{H}=\left(\zeta_{x}, \zeta_{p}\right)$. We need to solve for $X_{H}$ from the equation $d H . \xi=\omega\left(X_{H}, \xi\right)$. Now

$$
d H . \xi=<[p, x],[\delta p, x]>+<[p, x],[p, \delta x]>+<\frac{\partial V}{\partial x}, \delta x>
$$

and

$$
\omega\left(X_{H}, \xi\right)=<\zeta_{x}, \delta p>-<\zeta_{p}, \delta x>.
$$

Equating these expressions gives the result. 
Observe that for $V=0$ the Eqs. 2.2 are remarkably symmetric. Their symmetry with respect to $x$ and $p$ may in fact be expressed as follows: the equations (and the Hamiltonian) are invariant under the canonical transformation to new variables $X_{i}$ and $P_{i}$ where $X_{i}=p_{i}$ and $P_{i}=-x_{i}$. This transformation is generated by the generating function $S=\sum x_{k} X_{k}$ (see, for example, Goldstein [1980] or Abraham and Marsden [1978].) The high degree of symmetry of these equations is of course reflected in the geometric and integrability properties of the equations discussed below. (See also Howe [1984] for a discussion of symmetrical systems.)

We now consider flows of the above type on the cotangent bundle of an orbit. We have

Lemma 2.2. The natural cotangent bundle symplectic structure on $T^{*} \mathcal{O}$ for tangent vectors $X_{1}, X_{2} \in T T^{*} \mathcal{O}$ given by

$$
\begin{aligned}
& X_{1}=\left(x, \operatorname{ad}_{x}^{*} \xi,\left[x, \eta_{1}\right], \operatorname{ad}_{x}^{*} \zeta_{1}+\operatorname{ad}_{\left[x, \eta_{1}\right]}^{*} \xi\right), \\
& X_{2}=\left(x, \operatorname{ad}_{x}^{*} \xi,\left[x, \eta_{2}\right], \operatorname{ad}_{x}^{*} \zeta_{2}+\operatorname{ad}_{\left[x, \eta_{2}\right]}^{*} \xi\right),
\end{aligned}
$$

is

$$
\omega\left(X_{1}, X_{2}\right)=<\left[x, \eta_{1}\right],\left[x, \zeta_{2}\right]>-<\left[x, \eta_{2}\right],\left[x, \zeta_{1}\right]>+2<\left[\xi,\left[x, \eta_{1}\right]\right],\left[x, \eta_{2}\right]>.
$$

Proof. Note that the symplectic structure here is on the cotangent bundle of the orbit, in contrast to the orbit form which is defined on the orbit itself (see e.g. Abraham and Marsden [1978] or Arnold [1978]).

We recall that for any manifold $M$ the canonical symplectic form on $T^{*} M$ is defined as follows (see e.g. Arnold [1978] or Abraham and Marsden [1978]). Let $X \in T\left(T^{*} M\right)_{p}$ be a vector tangent to $T^{*} M$ at the point $p \in T^{*} M_{x}$. The derivative $\pi_{*}: T\left(T^{*} M\right) \rightarrow T M$ of the natural projection $\pi: T^{*} M \rightarrow M$ takes $X$ to a vector $\pi_{*} X$ tangent to $M$ at $x$. Define the one-form $\theta$ on $T^{*} M$ by $\theta(X)=p\left(\pi_{*} X\right)$. Define the symplectic form $\omega$ by $\omega=d \theta$.

Now let $M=\mathcal{O} \subset \mathfrak{g}$. Let $<,>$ be the natural pairing between elements of $\mathfrak{g}$ and $\mathfrak{g}^{*}$ given by the Killing form. Elements of $T_{x} \mathcal{O}$ are given by $[x, \eta], x, \eta \in \mathfrak{g}$ and elements of $T_{x}^{*} \mathcal{O}$ are thus determined by the form $<\xi,[x, \eta]>$. Or, if we set $<\xi,[x, \eta]>=$ $<\operatorname{ad}_{x}^{*} \xi, \eta>$, we may denote points of $T^{*} \mathcal{O}$ by pairs $\left(x, \operatorname{ad}_{x}^{*} \xi\right), x \in \mathfrak{g}, \xi \in \mathfrak{g}^{*}$.

To obtain points of $T T^{*} \mathcal{O}$ consider

$$
\begin{aligned}
\left.\frac{d}{d t}\right|_{t=0}\left(e^{-\eta t} x e^{\eta t},<\xi(t),\left[e^{-\eta t} x e^{\eta t}, \cdot\right]>\right) & =([x, \eta],<\zeta,[x, \cdot]>+<\xi,[[x, \eta], \cdot]> \\
& \sim\left([x, \eta], \operatorname{ad}_{x}^{*} \zeta+\operatorname{ad}_{[x, \eta]}^{*} \xi\right)
\end{aligned}
$$

where $\zeta=\xi^{\prime}(0)$ and $x, \eta \in \mathfrak{g}, \zeta, \xi \in \mathfrak{g}^{*}$.

Hence elements of $T\left(T^{*} \mathcal{O}\right)$ may be written

$$
\left(x, \operatorname{ad}_{x}^{*} \xi,[x, \eta], \operatorname{ad}_{x}^{*} \zeta+\operatorname{ad}_{[x, \eta]}^{*} \xi\right) .
$$

Now we have

$$
\pi: T^{*} \mathcal{O} \rightarrow \mathcal{O}:\left(x, \operatorname{ad}_{x}^{*} \xi\right) \rightarrow x
$$

and

$$
\pi^{*}: T\left(T^{*} \mathcal{O}\right) \rightarrow T \mathcal{O}:\left([x, \eta], \operatorname{ad}_{x}^{*} \zeta+\operatorname{ad}_{[x, \eta]}^{*} \xi\right) \rightarrow[x, \eta]
$$


Hence for an arbitrary element $X$ of $T\left(T^{*} \mathcal{O}\right)$ we have

$$
\begin{aligned}
\theta\left(\pi^{*} X\right) & =\operatorname{ad}_{x}^{*} \xi([x, \eta]) \\
& \sim<\operatorname{ad}_{x}^{*} \xi,[x, \eta]> \\
& =<[\xi, x],[x, \eta]>.
\end{aligned}
$$

Now the canonical symplectic form is given by

$$
\omega=d \theta=d<[\xi, x], \cdot>\text {. }
$$

Computing the exterior derivative $d$ in local cordinates, for $X_{1}, X_{2} \in T T^{*} \mathcal{O}$, where

$$
\begin{aligned}
& X_{1}=\left(x, \operatorname{ad}_{x}^{*} \xi,\left[x, \eta_{1}\right], \operatorname{ad}_{x}^{*} \zeta_{1}+\operatorname{ad}_{\left[x, \eta_{1}\right]}^{*} \xi\right), \\
& X_{2}=\left(x, \operatorname{ad}_{x}^{*} \xi,\left[x, \eta_{2}\right], \operatorname{ad}_{x}^{*} \zeta_{2}+\operatorname{ad}_{\left[x, \eta_{2}\right]}^{*} \xi\right),
\end{aligned}
$$

we have

$$
\begin{aligned}
\omega\left(X_{1}, X_{2}\right)= & \left(\operatorname{ad}_{x}^{*} \zeta_{1}+\operatorname{ad}_{\left[x, \eta_{1}\right]}^{*} \xi\right)\left(\left[x, \eta_{2}\right]\right)-\left(\operatorname{ad}_{x}^{*} \zeta_{2}+\operatorname{ad}_{\left[x, \eta_{2}\right]}^{*} \xi\right)\left(\left[x, \eta_{1}\right]\right) \\
= & <\left[\zeta_{1}, x\right],\left[x, \eta_{2}\right]>+<\left[\xi,\left[x, \eta_{1}\right]\right],\left[x, \eta_{2}\right]> \\
& -<\left[\zeta_{2}, x\right],\left[x, \eta_{1}\right]>-<\left[\xi,\left[x, \eta_{2}\right]\right],\left[x, \eta_{1}\right]> \\
= & <\left[x, \eta_{1}\right],\left[x, \zeta_{2}\right]>-<\left[x, \eta_{2}\right],\left[x, \zeta_{1}\right]>+2<\left[\xi,\left[x, \eta_{1}\right]\right],\left[x, \eta_{2}\right]>,
\end{aligned}
$$

as required.

We now have

Theorem 2.3. The equations

$$
\begin{aligned}
\dot{x} & =[x,[p, x]] \\
\dot{p} & =[p,[p, x]] .
\end{aligned}
$$

are the Hamiltonian form of the geodesic equations on an adjoint orbit of $\mathfrak{g}_{\mathfrak{u}}$.

The structure of the proof is as follows: first we consider the structure of the Hamiltonian and then we derive the explicit form of the Hamiltonian flow using the lemma above.

For $V=0$ in (2.1) we shall show that the Hamiltonian is just the norm of the velocity in the so-called normal metric (see e.g. Bloch, Brockett and Ratiu [1992]). This metric is defined as follows: Let $x$ and $l$ lie in $\mathfrak{g}_{\mathfrak{u}}$. Then $\mathrm{x}$ may be decomposed as $x=x^{l}+x_{l}$ where $x_{l} \in \operatorname{Ker}\left(\operatorname{ad}_{l}\right)$ and $x^{l} \in \operatorname{Im}\left(\operatorname{ad}_{l}\right)$ and where $\operatorname{ad}_{x}(y)=[x, y]$. Further, given any $l \in \mathfrak{g}_{\mathfrak{u}}$ we may decompose $\mathfrak{g}_{\mathfrak{u}}$ orthogonally relative to $-\kappa($,$) as \mathfrak{g}_{\mathfrak{u}}^{\mathfrak{l}} \oplus \mathfrak{g}_{\mathfrak{u} \mathfrak{l}}$ where $\mathfrak{g}_{\mathfrak{u}}^{\mathfrak{l}}=\operatorname{Im}\left(\mathrm{ad}_{\mathfrak{l}}\right)$ and $\mathfrak{g}_{\mathfrak{u l}}=\operatorname{Ker}\left(\operatorname{ad}_{\mathfrak{l}}\right)$. Now if $\xi=[x, a]$ and $\eta=[x, b]$ are tangent vectors to the orbit at $x$ then the normal metric is given by $g_{n}(\xi, \eta)=-\kappa\left(a^{x}, b^{x}\right)$.

Now any velocity vector is tangent to the orbit of the adjoint action and hence is of the form $\dot{x}=[x, a]$ for some $x \in \mathfrak{g}_{\mathfrak{u}}$. Hence $\dot{x} \in \operatorname{Im}\left(\operatorname{ad}_{l}\right)$. The inverse of operator $\operatorname{ad}_{x}$, we will denote by $\operatorname{ad}_{x}^{-1}$, is well defined on $\operatorname{Im}\left(\operatorname{ad}_{x}\right)$ and hence on $\dot{x}$.

The kinetic energy may thus be written:

$$
L(x, \dot{x})=1 / 2<\dot{x}^{x}, \dot{x}^{x}>=1 / 2\left(\|\left[\operatorname{ad}_{x}^{-1} \dot{x} \|^{2}\right) .\right.
$$

Then, since $1 / 2\left\|\operatorname{ad}_{x}^{-1} \dot{x}\right\|^{2}=-1 / 2<\operatorname{ad}_{x}^{-2} \dot{x}, \dot{x}>$ we have, applying the Legendre transform, 


$$
p=\frac{\partial L}{\partial \dot{x}}=-\operatorname{ad}_{x}^{-2} \dot{x} .
$$

Substituting this expression for $p$ into (2.8) gives the required expression for the kinetic energy.

It remains to show that while these equation are Hamiltonian with respect to the standard symplectic structure on $T^{*} \mathfrak{g}_{\mathfrak{u}}$, they are in fact Hamiltonian on the cotangent bundle of an adjoint orbit of $G_{u}$. That is, even though the Hamiltonian structure on the orbit is complicated and is not the restriction of the structure on the Lie algebra, the equations themselves do restrict.

We remark that the Eqs. 2.7 may be viewed intrinsically as equations evolving in a Lie bialgebra - see e.g. Lu and Weinstein [1990]. We shall discuss this and related ideas further in a forthcoming publication.

For ease of exposition we now identify $T\left(T^{*} \mathcal{O}\right)$ with $T(T \mathcal{O})$ via the metric induced by the Killing form.

Elements of $T T_{x,[x, \xi]} \mathcal{O}$ are thus of the form

$$
(x,[x, \xi],[x, \eta],[[x, \eta], \xi]+[x, \zeta])
$$

for $\xi, \eta, \zeta \in \mathfrak{g}_{\mathfrak{u}}$, and for any two such elements $X_{1}, X_{2}, \omega\left(X_{1}, X_{2}\right)$ is given by (2.3).

We now wish to compute the Hamiltonian flow of the Hamiltonian

$$
H=\frac{1}{2}\|[p, x]\|^{2}=\frac{1}{2}<\operatorname{ad}_{x}^{-1} \dot{x}, \operatorname{ad}_{x}^{-1} \dot{x}>=\frac{1}{2}<\dot{x}^{x}, \dot{x}^{x}>
$$

- the kinetic energy in the normal metric. For this we need to solve for $X_{H}$ from the expression

$$
d H . \delta X=\omega\left(X_{H}, \delta X\right)
$$

for $\delta X$ an arbitrary element of $T T \mathcal{O}$. Setting

$$
\begin{aligned}
\delta X & =\left(x,[x, \xi],\left[x, \eta_{1}\right],\left[\left[x, \eta_{1}\right], \xi\right]+\left[x, \zeta_{1}\right]\right), \\
X_{H} & =\left(x,[x, \xi],\left[x, \eta_{2}\right],\left[\left[x, \eta_{2}\right], \xi\right]+\left[x, \zeta_{2}\right]\right),
\end{aligned}
$$

$\omega\left(X_{H}, \delta X\right)$ is given by the expression 2.3 .

We now need to compute $d H . \delta X$ employing variations in $T_{x,[x, \xi]} T \mathcal{O}$. We observe firstly that

$$
\delta \operatorname{ad}_{x}^{-1} \dot{x}=-\operatorname{ad}_{x}^{-1}\left[\delta x, \operatorname{ad}_{x}^{-1} \dot{x}\right]+\operatorname{ad}_{x}^{-1} \delta \dot{x} .
$$

This follows from setting

$$
\operatorname{ad}_{x}\left(\operatorname{ad}_{x}^{-1}(c)\right)=c
$$

for $c$ a constant. For then

$$
\left[\delta x, \operatorname{ad}_{x}^{-1}(c)\right]+\left[x, \delta \operatorname{ad}_{x}^{-1}(c)\right]=0
$$

or

$$
\delta \operatorname{ad}_{x}^{-1}(c)=-\operatorname{ad}_{x}^{-1}\left[\delta x, \operatorname{ad}_{x}^{-1}(c)\right] .
$$

Replacing $c$ by $\dot{x}$ gives the result.

Now 


$$
\begin{aligned}
d H . \delta X= & <\operatorname{ad}_{x}^{-1} \dot{x},-\operatorname{ad}_{x}^{-1}\left[\delta x, \operatorname{ad}_{x}^{-1} \dot{x}\right]+\operatorname{ad}_{x}^{-1} \delta \dot{x}> \\
= & <\operatorname{ad}_{x}^{-1} \dot{x},-\operatorname{ad}_{x}^{-1}\left[\left[x, \eta_{1}\right], \operatorname{ad}_{x}^{-1} \dot{x}\right]+\operatorname{ad}_{x}^{-1}\left(\left[\left[x, \eta_{1}\right], \xi\right]+\left[x, \zeta_{1}\right]\right)> \\
= & <\left[x, \operatorname{ad}_{x}^{-2} \dot{x}\right],-\operatorname{ad}_{x}^{-1}\left[\left[x, \eta_{1}\right], \operatorname{ad}_{x}^{-1} \dot{x}\right]+\operatorname{ad}_{x}^{-1}\left(\left[\left[x, \eta_{1}\right], \xi\right]+\left[x, \zeta_{1}\right]\right)> \\
= & <\operatorname{ad}_{x}^{-2} \dot{x},\left[\left[x, \eta_{1}\right], \operatorname{ad}_{x}^{-1} \dot{x}\right]-\left[\left[x, \eta_{1}\right], \xi\right]+\left[x, \zeta_{1}\right]> \\
= & \left.<\operatorname{ad}_{x}^{-1} \dot{x}, \operatorname{ad}_{x}^{-2} \dot{x}\right],\left[x, \eta_{1}\right]>-<\left[\xi, \operatorname{ad}_{x}^{-2} \dot{x}\right],\left[x, \eta_{1}\right]> \\
& -<\operatorname{ad}_{x}^{-2} \dot{x},\left[x, \zeta_{1}\right]> \\
= & -<\operatorname{ad}_{x}^{-2} \dot{x},\left[x, \zeta_{1}\right]>,
\end{aligned}
$$

the last inequality holding since $\xi=\operatorname{ad}_{x}^{-1} \dot{x}$, and thus the first two terms cancel.

Now equating the coefficients of $\left[x, \eta_{1}\right]$ and $\left[x, \zeta_{1}\right]$ in $(2.14)$ and $(2.3)$ we get

$$
\begin{aligned}
& 2\left[\xi,\left[x, \eta_{2}\right]\right]-\left[x, \zeta_{2}\right]=0, \\
& {\left[x, \eta_{2}\right]=\operatorname{ad}_{x}^{-2} \dot{x}=-p .}
\end{aligned}
$$

Note also that since $\xi=\operatorname{ad}_{x}^{-1} \dot{x}$ or $\xi=\left[x, \operatorname{ad}_{x}^{-2} \dot{x}\right]$ we have

$$
\xi=[p, x] \text {. }
$$

The final term in the equation for $X_{H}$ is thus

$$
\begin{aligned}
{\left[\left[x, \eta_{2}\right], \xi\right]+\left[x, \zeta_{2}\right] } & =\left[\left[x, \eta_{2}\right], \xi\right]+2\left[\xi,\left[x, \eta_{2}\right]\right] \\
& =-[p,[p, x]]+2[[p, x],-p] \\
& =[p,[p, x]] .
\end{aligned}
$$

Hence the full expression for $X_{H}$ is

$$
(x,[x,[p, x]],-p,[p,[p, x]]) .
$$

We also have

Theorem 2.4. The Hamiltonian flows of $f([p, x])=f(\xi)$, where $f$ is an invariant polynomial on $\mathfrak{g}_{\mathfrak{u}}$ are given by:

$$
\begin{aligned}
& \dot{x}=[x,[p, x]] \\
& \dot{p}=\left[-\operatorname{ad}_{x}^{-1} f^{\prime}([p, x]),[p, x]\right] .
\end{aligned}
$$

Proof. In this case we have, using the same notation as in the previous theorem,

$$
\begin{aligned}
d f(\xi) . \delta X & =<f^{\prime}(\xi), \delta X> \\
& =<f^{\prime}(\xi), \delta X>.
\end{aligned}
$$

Computing as in the previous theorem we obtain

$$
\left[x, \eta_{2}\right]=\operatorname{ad}_{x}^{-1} f^{\prime}(\xi)
$$

and

$$
\begin{aligned}
{\left[\left[x, \eta_{2}\right], \xi\right]+\left[x, \zeta_{2}\right] } & =\left[\xi,\left[x, \eta_{2}\right]\right] \\
& =\left[\xi, \operatorname{ad}_{x}^{-1} f^{\prime}(\xi)\right]
\end{aligned}
$$

so

$$
X_{f}=\left(x,[x,[p, x]], \operatorname{ad}_{x}^{-1}\left(f^{\prime}([p, x]),\left[[p, x], \operatorname{ad}_{x}^{-1} f^{\prime}([p, x])\right]\right) .\right.
$$


We may also endow any orbit with the left invariant metric

$$
g_{n l}([x, a],[x, b])=-\kappa\left(a^{x}, J b^{x}\right),
$$

where $\mathbf{J}$ is a positive self-adjoint operator on the algebra. Then we have

Corollary 2.5. The geodesic equations on an adjoint orbit endowed with the left invariant metric (2.18) are

$$
\begin{aligned}
\dot{x} & =\left[x, J^{-1}[p, x]\right]=-\left[x, J^{-1}[x, p]\right], \\
\dot{p} & =\left[p, J^{-1}[p, x] .\right.
\end{aligned}
$$

Proof. The kinetic energy in this case is given by

$$
\begin{aligned}
L(x, \dot{x}) & =1 / 2<\dot{x}^{x}, \dot{x}^{x}> \\
& =1 / 2\left(-<\operatorname{ad}_{x}^{-2} \dot{x},\left[x, \operatorname{Jad}_{x}^{-1} \dot{x}\right]>\right. \\
& =-1 / 2<\dot{x}, \operatorname{ad}_{x}^{-1} \operatorname{Jad}_{x}^{-1} \dot{x}>.
\end{aligned}
$$

Hence

$$
p=-\operatorname{ad}_{x}^{-1} \operatorname{Jad}_{x}^{-1} \dot{x},
$$

and

$$
[p, x]=J_{\mathrm{ad}_{x}^{-1} \dot{x}}
$$

Thus the kinetic energy may be reexpressed as

$$
\frac{1}{2}<J^{-1}[p, x],[p, x]>
$$

From now on however unless otherwise stated we shall consider the biinvariant case. We shall in fact consider left (or right) invariant metrics only when we study the generalized rigid body flows below.

We remark also that if we define $z=x+i p$, then Eqs. 2.7 may also be written

$$
\dot{z}=\left[z,\left[\bar{z}, \frac{i z}{2}\right]\right]
$$

the form of an interesting class of Hamiltonian flows discussed in Brockett [1993]. 


\section{Flows on Symmetric Spaces}

The equations discussed above are not only well defined on adjoint orbits but also on general symmetric spaces where the tangent vectors to the space are given in the form a suitable bracket - this includes the complex and real Grassmannians of q-planes in $n+1$-space $G_{q, n+1}(\mathbb{C})$ or $G_{q, n+1}(\mathbb{R})$ and in particular the spheres.

This may be seen as follows:

The complex Grassmannian is given by

$$
U(n+1) / U(q) \times U(p), \quad q+p=n+1, q \leq p
$$

and the real Grassmannian by

$$
S O(n+1) / S O(q) \times S O(p), \quad q+p=n+1, q \leq p,
$$

where $U(n)$ is the unitary group and $S O(n)$ the special orthogonal group. In either case let $\mathfrak{g}=\mathfrak{k} \oplus \mathfrak{m}$ be the Lie algebra decomposition corresponding to $G / K$. We may thus represent a point in the complex (real) Grassmannian by a matrix

$$
\hat{Q}=\left[\begin{array}{ll}
0 & Q \\
-Q^{*} & 0
\end{array}\right]
$$

in $\mathfrak{m}$ where $Q$ is a complex (real) $p \times q$ matrix of full rank and $Q^{*}$ is its Hermitian conjugate (transpose). A point in $\mathfrak{k}$ may be represented by the matrix

$$
\hat{K}=\left[\begin{array}{ll}
K_{1} & O \\
O & K_{2}
\end{array}\right],
$$

where $K_{1} \in u(p)(s o(p))$ and $K_{2} \in u(q)(s o(q))$. Define $\hat{P}$ to be a similarly partitioned matrix. Then we have

Proposition 3.1. Tangent vectors to the Grassmannian may be represented by matrices of the form

$$
[\hat{Q}, \hat{K}]
$$

Proof. A curve in the Grassmannian through the point $\hat{Q}$ may be given by

$$
e^{-\hat{K} t} \hat{Q} e^{\hat{K} t}
$$

Note that the given curve simply provides an orthogonal (or unitary) transformation of the rows and columns of $Q$.

Differentiating at $t=0$ gives the result.

Now since tangent vectors are given by brackets, just as in the case of orbits, a normal metric may be defined. Repeating the proof of Theorem 2.2 gives

Proposition 3.2. The geodesic equations on the real or complex Grassmannian are given by

$$
\begin{aligned}
& \dot{\hat{Q}}=[\hat{Q},[\hat{P}, \hat{Q}]], \\
& \dot{\hat{P}}=[\hat{P},[\hat{P}, \hat{Q}]],
\end{aligned}
$$

where $\hat{Q}$ is given by (3.25) and similarly for $\hat{P}$. 
Note also that for a symmetric space $[\mathfrak{k}, \mathfrak{k}] \subset \mathfrak{k},[\mathfrak{k}, \mathfrak{m}] \subset \mathfrak{m}$ and $[\mathfrak{m}, \mathfrak{m}] \subset \mathfrak{k}$ and since $Q, P \in \mathfrak{m}$ the equations are naturally well defined.

Further, we have

Corollary 3.3. For a right invariant "normal" metric on the Grassmannian the geodesic flow is given by the double double bracket equations

$$
\begin{aligned}
& \dot{\hat{Q}}=\left[\hat{Q}, J^{-1}[\hat{P}, \hat{Q}]\right], \\
& \dot{\hat{P}}=\left[\hat{P}, J^{-1}[\hat{P}, \hat{Q}]\right],
\end{aligned}
$$

where $J=\left[J_{U}, J_{L}\right]$ is a symmetric positive definite operator partioned in accordance with $\hat{Q}$ and $\hat{P}$.

The proof is a computation.

We also have

Corollary 3.4. Equations 3.28 are Hamiltonian on $T^{*} s u(n+1)\left(T^{*} s o(n+1)\right)$ and on $T^{*} G_{q, n+1}(\mathbb{C})\left(T^{*} G_{q, n+1}(\mathbb{R})\right)$ with respect to the standard cotangent bundle symplectic form in each case and with Hamiltonian

$$
H=\frac{1}{4}<[\hat{P}, \hat{Q}], J^{-1}[\hat{P}, \hat{Q}]>
$$

As an example in the current setting we write explicitly the geodesic flow on the sphere $S^{n}$.

Recall (see e.g. Moser [1980]) that the geodesic motion on $S^{n}$ may be written as follows:

Let $\mathbf{q}=\left[q_{1}, \cdots, q_{n+1}\right]^{T} \in \mathbb{R}^{n+1}$ with Euclidean norm $\|\mathbf{q}\|=1$ represent an element of $S^{n}$. Then the geodesic flow can be found by setting $\ddot{\mathbf{q}}=\lambda \mathbf{q}$ where $\lambda$ is chosen so that $\|\mathbf{q}\|$ is compatible with the flow. This implies $<\mathbf{q}, \dot{\mathbf{q}}>=0$ and $<\mathbf{q}, \ddot{\mathbf{q}}>+\|\dot{\mathbf{q}}\|^{2}=0$. Thus $\lambda=-\|\dot{\mathbf{q}}\|^{2}$ and the geodesic flow is given by

$$
\ddot{\mathbf{q}}=-\|\dot{\mathbf{q}}\|^{2} \mathbf{q} \text {. }
$$

Letting $\mathbf{p}=\left[p_{1}, \cdots, p_{n+1}\right]^{T} \in \mathbb{R}^{n+1}$ this may be viewed as a Hamiltonian system restricted to $\|\mathbf{q}\|=1,<\mathbf{q}, \mathbf{p}>=0$. With Hamiltonian $H=1 / 2\|\mathbf{q}\|^{2}\|\mathbf{p}\|^{2}$ we get the flow

$$
\dot{\mathbf{q}}=\left(\frac{\partial H}{\partial \mathbf{p}}\right)^{T}=\mathbf{p} \quad \dot{\mathbf{p}}=-\left(\frac{\partial H}{\partial \mathbf{q}}\right)^{T}=-\|\mathbf{p}\|^{2} \mathbf{q} .
$$

In our current setting we have

Proposition 3.5. Let

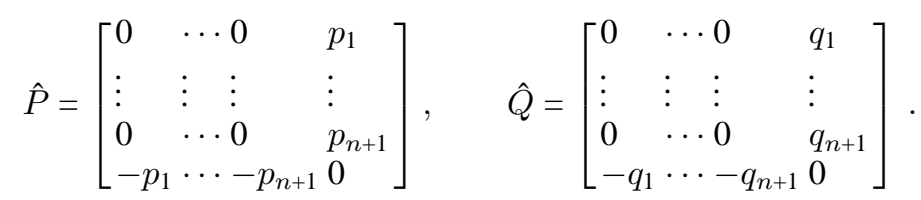

Then the flow (3.27) restricts to the geodesic flow (3.30). 
Proof. The proof is essentially a computation. Computing the brackets gives

$$
\begin{aligned}
& \dot{\mathbf{q}}=\mathbf{p}\left(\mathbf{q}^{T} \mathbf{q}\right)-\left(\mathbf{q}^{T} \mathbf{p}\right) \mathbf{q}, \\
& \dot{\mathbf{p}}=\left(\mathbf{q}^{T} \mathbf{p}\right) \mathbf{p}-\mathbf{q}\left(\mathbf{p}^{T} \mathbf{p}\right) .
\end{aligned}
$$

It is easy to check that the time derivatives of $\mathbf{q}^{T} \mathbf{q}$ and $\mathbf{q}^{T} \mathbf{p}$ are conserved along the flow. Normalizing $\|\mathbf{q}\|=1$ and $\langle\mathbf{q}, \mathbf{p}>=0$ we indeed remain on the sphere.

Note that in this case (3.29) gives

$$
H=\frac{1}{2}\left(<\mathbf{p}, \mathbf{p}><\mathbf{q}, \mathbf{q}>-<\mathbf{q}, \mathbf{p}>^{2}>\right) .
$$

This gives rise to Eq. 3.33 via the standard symplectic structure on $\mathbb{R}^{2 n+2}$ and restricts to the geodesic flow on the sphere when we impose the constraints $\|\mathbf{q}\|=1$ and $<\mathbf{q}, \mathbf{p}>=0$.

\section{The Rigid Body Equations}

We show here how the generalized rigid body equations, another well known integrable system, may be written in double double bracket form.

We recall the rigid body equations on $S O(n)$ (or generally on any compact Lie group - see e.g. Abraham and Marsden [1978], Ratiu [1980]) may be written as

$$
\begin{aligned}
\dot{Q} & =\Omega Q, \\
\dot{M} & =[\Omega, M],
\end{aligned}
$$

where $Q \in S O(n)$ denotes the configuration space variables, $\Omega \in s o(n)$ is the angular velocity, and $M=J \Omega=\Lambda \Omega+\Omega \Lambda$ is the angular momentum. Here $J$ is a symmetric positive definite operator defined by the diagonal positive definite matrix $\Lambda$. We remark that the rigid body equations here are written in right-invariant as opposed to the commonly used left-invariant form in order to be consistent with the conventions used in the remainder of the paper. This results in a sign change in the second of Eqs. 4.35.

The classical rigid body Eqs. 4.35 are of course Hamiltonian on $T^{*} S O(n)$ with respect to the canonical symplectic structure.

We now consider the following equations:

$$
\begin{aligned}
& \dot{Q}=\Omega Q, \\
& \dot{P}=\Omega P,
\end{aligned}
$$

where $\Omega=J^{-1} M$ and $M=P Q^{T}-Q P^{T}$, where $Q$ and $P$ are in $S O(n)$.

We then can easily check that

Proposition 4.1. Equations 4.36 reduce to the rigid body Eqs. 4.35.

Proof. Differentiating $M=P Q^{T}-Q P^{T}$ and using the Eqs. 4.36 gives the second of Eqs. 4.35. 
Conversely, given the rigid body Eqs. 4.35 we may solve for the variable $P$ in the expression

$$
M=P Q^{T}-Q P^{T}
$$

in a neighborhood of $M=0$. Locally

$$
P=\left(e^{\sinh ^{-1} M / 2}\right) Q
$$

This follows from the observation that

$$
M=e^{\sinh ^{-1} M / 2}-e^{-\sinh ^{-1} M / 2} .
$$

For $s o(n)$ however sinh is many to one, so the two representations are not entirely equivalent. Note that the generalized rigid body flow naturally reduces to a flow in the variables $M$ on an adjoint orbit of $s o(n)$ and we can view the map which takes $P Q^{T}-Q P^{T}$ to $M$ as reduction. In fact, the map $(Q, P) \rightarrow(Q, M)$ given above is a canonical transformation from the symplectic structure on $T^{*} g l(n)$ to that on $T^{*} S O(n)$ which intertwines the Hamiltonian equations 4.36 on $T^{*} g l(n)$ with the Hamiltonian Eqs. 4.35 on $T^{*} S O(n)$.

While the classical rigid body Eqs. 4.35 are Hamiltonian on $T^{*} S O(n)$ with respect to the canonical symplectic structure on the group in contrast here we have

Proposition 4.2. The generalized rigid body equations in the form (4.36) are Hamiltonian on $T^{*} g l(n)$ with respect to the canonical symplectic structure and the Hamiltonian

$$
H=1 / 4<J^{-1}\left(P Q^{T}-Q P^{T}\right), P Q^{T}-Q P^{T}>.
$$

We remark that here $P$ and $Q$ are natural coordinates for $T^{*} g l(n)$ and, for $P(0)$, $Q(0) \in S O(n), P(t)$ and $Q(t)$ evolve in $S O(n)$ under the flow of $H$. Hence $S O(n) \times$ $S O(n)$ is an invariant manifold for the flow of $H$. Note also that this Hamiltonian is equivalent to $H=1 / 4<J^{-1} M, M>$ as in Ratiu [1980].

However, the canonical symplectic structure for the rigid body may in fact be recovered as a special case of our symplectic form (2.3) as we shall show below.

We first make the following observation: the rigid body equations may be given as a singular case of the double double bracket equations discussed earlier.

Let

$$
\hat{Q}=\left[\begin{array}{ll}
0 & Q \\
-Q^{T} & 0
\end{array}\right]
$$

as before and similarly for $\hat{P}$. Note that these matrices now lie in $s o(2 n)$ and each block lies in $S O(n)$.

Corollary 4.3. The generalized rigid body equations on $S O(n)$ are given by the double double bracket Eqs. 3.28 in the case $Q$ and $P$ lie in $S O(n), J_{U}=J$, and the operator $J_{L}^{-1}=0$.

With the above in mind, for an arbitrary element of $s o(n), \mu$ say, define the following embedding into $\mathrm{sl}(2 \mathrm{n})$ :

$$
\tilde{\mu}=\left[\begin{array}{ll}
0 & \mu \\
0 & 0
\end{array}\right] \text {. }
$$

Then we have 
Lemma 4.4. An element of TTSO $(n)$ may be represented by

$$
X=(\hat{Q},[\tilde{\xi}, \hat{Q}],[\tilde{\Omega}, \hat{Q}],[\tilde{\xi},[\tilde{\Omega}, \hat{Q}]]+[\tilde{\zeta}, \hat{Q}]) .
$$

Proof. A (right invariant) element of $T S O(n)$ at the point $Q \in S O(n)$ may be written as $(Q, \xi Q)$. Differentiating a suitable one-parameter curve gives an element of $\operatorname{TTSO}(n)$ as

$$
(Q, \xi Q, \Omega Q, \zeta Q+\xi \Omega Q) .
$$

A matrix computation with the above embedding then gives the result.

Now identify $T^{*} S O(n)$ with $T S O(n)$ via the Killing form and use the following multiple of the Killing form as the inner product on elements of $s o(2 n):\langle\cdot, \cdot\rangle=$ $-1 / 2 \operatorname{Tr}(\cdot, \cdot)$, where $\operatorname{Tr}$ is the trace.

We have

Theorem 4.5. Let two vectors in $T T S O(n)$ be given by

$$
\begin{aligned}
& X_{1}=\left(\hat{Q},[\tilde{\xi}, \hat{Q}],\left[\tilde{\Omega}_{1}, \hat{Q}\right],\left[\tilde{\xi},\left[\tilde{\Omega}_{1}, \hat{Q}\right]\right]+\left[\tilde{\zeta}_{1}, \hat{Q}\right]\right), \\
& X_{2}=\left(\hat{Q},[\tilde{\xi}, \hat{Q}],\left[\tilde{\Omega}_{2}, \hat{Q}\right],\left[\tilde{\xi},\left[\tilde{\Omega}_{2}, \hat{Q}\right]\right]+\left[\tilde{\zeta}_{2}, \hat{Q}\right]\right) .
\end{aligned}
$$

Then the canonical symplectic form on $T^{*} S O(n) \sim T S O(n)$ is given by $\omega\left(X_{1}, X_{2}\right)$ with $\omega$ defined by (2.3).

Proof. From (2.3) we have in this case

$\omega\left(X_{1}, X_{2}\right)=<\left[\hat{Q}, \tilde{\Omega}_{1}\right],\left[\hat{Q}, \tilde{\zeta}_{2}\right]>-<\left[\hat{Q}, \tilde{\Omega}_{2}\right],\left[\hat{Q}, \tilde{\zeta}_{1}\right]>+2<\left[\tilde{\zeta},\left[\hat{Q}, \tilde{\Omega}_{1}\right]\right],\left[\hat{Q}, \tilde{\Omega}_{2}\right]>$.

Now, compute each term of this expression using the Trace and define the inner product on $s o(n)$ to be given by $\langle\cdot, \cdot\rangle=-\operatorname{Tr}(\cdot, \cdot)$. We find

$$
\begin{aligned}
<\left[\hat{Q}, \tilde{\Omega}_{1}\right],\left[\hat{Q}, \tilde{\zeta}_{2}\right]> & =-\operatorname{Tr} \Omega_{1} \zeta_{2}=<\Omega_{1}, \zeta_{2}>, \\
<\left[\hat{Q}, \tilde{\Omega_{2}}\right],\left[\hat{Q}, \tilde{\zeta}_{1}\right]> & =-\operatorname{Tr} \Omega_{2} \zeta_{1}=<\Omega_{2}, \zeta_{1}>, \\
<\left[\tilde{\zeta},\left[\hat{Q}, \tilde{\Omega_{1}}\right]\right],\left[\hat{Q}, \tilde{\Omega_{2}}\right]> & =\frac{1}{2}<\xi,\left[\Omega_{1}, \Omega_{2}\right]>.
\end{aligned}
$$

Thus

$$
\omega\left(X_{1}, X_{2}\right)=<\Omega_{1}, \zeta_{2}>-<\Omega_{2}, \zeta_{1}>+<\xi,\left[\Omega_{1}, \Omega_{2}\right]>
$$

This is precisely the canonical form on $T^{*} S O(n)$ (see e.g. Abraham and Marsden [1978]).

Note that the reduced generalized rigid body equations (the dynamics, or second of Eqs. 4.35 are completely integrable (see e.g. Ratiu [1980] and references therein). In fact the full system is integrable in the sense that the reduced phase space is a point, see Abraham and Marsden [1978], and hence also in the sense of commutative integrability discussed in the next section (see Mischenko and Fomenko [1978]). The full phase space is $M=T^{*} S O(n)$, on which we have a natural action of the group $G=S O(n)$. The 
reduced phase space corresponding to this is $M_{\mu}=J^{-1}(\mu) / G_{\mu}$, where $J$ here is the momentum map corresponding to the action of $G$ on $M$ and $G_{\mu}$ is the stabilizer of the coadjoint action of $G$ on its Lie algeba. $M_{\mu}$ has $\operatorname{dimension} \operatorname{dim} M-\operatorname{dim} G-\operatorname{dim} G_{\mu}$ and is symplectic. On $M_{\mu}$ the system is then Liouville or commutatively completely integrable.

\section{Integrability of the Geodesic Flow on Grassmannians}

To prove (commutative) complete integrability of a Hamiltonian system on a symplectic manifold of dimension $2 n$ one needs of course to show that there are $n$ independent integrals in involution on the manifold. Thimm [1981] showed there were sufficiently many independent integrals in involution for the geodesic flows on the real and complex Grassmannians to be integrable.

In fact one can make a very general argument regarding integrability as follows: see Guillemin and Sternberg [1983,84] (and Paternain and Spatzier [1994]) for an account of this work. Suppose one has the Hamiltonian action of a Lie group $G$ on a a symplectic manifold $M$. The action is said to be multiplicity free if all G-invariant functions on $M$ commute under the Poisson bracket. Consider an ascending chain of Lie algebras $\mathfrak{g}_{\mathfrak{i}}$

$$
\mathfrak{g}_{1} \subset \mathfrak{g}_{2} \cdots \subset \mathfrak{g}_{\mathfrak{n}+1}=\mathfrak{g}_{\mathfrak{u}}
$$

with corresponding Lie Groups $G_{i}$. Each subgroup $G_{i}$ acts via a Hamiltonian action on coadjoint orbits of $G_{i_{1}}$ in $\mathfrak{g}_{\mathfrak{i}}$ (equipped with the orbit symplectic structure). The chain $G_{i}$ is said to be multiplicity free if all such actions are multiplicity-free. For compact groups this forces the $G_{i}$ to be locally isomorphic to the special orthogonal or unitary groups, tori or products of these. If one has such a chain and a multiplicity free action on $M$ then any $G$-invariant Hamiltonian system on $N$ is integrable.

Particular cases to which this applies are the real and complex Grassmannians. Here we construct an explicit proof of integrability via the formalism developed above.

We remark that the terminology arises from the representation theory of Lie groups. If $G$ is a Lie group, then a unitary representation of $G$ on a Hilbert space $H$ is called multiplicity free if every representation of $G$ acts on $H$ with multiplicity zero or one. This is the case if and only if the ring of bounded $G$-invariant functions on $H$ is commutative.

Guillemin and Sternberg were led to the symplectic analogue of the representation theoretic ideas by studying so called collective completely integrable systems, i.e. systems where the integrals are of the form $f \circ \Phi$ where $\Phi: M \rightarrow \mathfrak{g}^{*}$ is the moment mapping and $f$ is a function on $\mathfrak{g}^{*}$. They noticed that a necessary condition for $M$ to admit such system is that the action of $G$ on $M$ be multiplicity free. Our integrals are also of this type (see below).

We need first some preliminary results:

Let $\pi_{k}$ denote the projection of an element of $\mathfrak{g}$ onto an element of $\mathfrak{g}_{\mathfrak{k}}$, as defined above. We consider the following sequence of integrals:

$$
f\left(\pi_{k}[p, x]\right),
$$

where $f \in C^{\infty}$ is an invariant polynomial on $\mathfrak{g}_{\mathfrak{i}}$.

We note that such integrals are also in fact functions of the momentum map arising from the lifted action of the algebra on the underlying manifold $M$ (orbit or symmetric space) to the cotangent bundle. 
For, let $\xi_{M}$ denote the infinitesimal vector field corresponding to the action of $e^{t \xi}$ on the manfold via conjugation. This is given by $\xi_{M}=[x, \xi]$. Then the momentum map of the lifted action is given by (see e.g. Marsden and Ratiu [1994])

$$
<\Phi\left(\alpha_{x}\right), \xi>=<\alpha_{x}, \xi_{M}>
$$

where $\alpha_{x}$ is a point in the cotangent bundle at the point $x$.

In our setting this equation becomes

$$
-<\left[x,[x, \alpha], \xi_{>}=<[x, \alpha],[x, \xi]>,\right.
$$

where $<[x, \alpha], \cdot>$ represents a cotangent vector. Hence the lifted momentum map is indeed of the $[x, p]$, where $p$ is a momentum vector.

Now let $\mathfrak{g}$ be $s o(n)$ or $s u(n)$. Then we have

Proposition 5.1. The Hamiltonian flow of the integrals 5.45 on $T^{*} \mathfrak{g}$ is given by

$$
\dot{x}=\left[x, f^{\prime}\left(\pi_{k}[p, x]\right)\right] \quad \dot{p}=\left[p, f^{\prime}\left(\pi_{k}[p, x]\right)\right] .
$$

Firstly we note the following lemma:

Lemma 5.2. For $a$ and $b \in \mathfrak{g}$ we have

$$
<\pi_{k} a, b>=<a, \pi_{k} b>
$$

and

$$
<\pi_{k} b,\left[f\left(\pi_{j} a\right), a\right]>=0, k \geq j,
$$

where $f$ is an invariant polynomial on $\mathfrak{g}_{\mathfrak{u}}$.

Proof. This follows by elementary matrix computation.

Proof of Proposition. Let $\xi=(\delta x, \delta p)$ denote an arbitrary tangent vector to $T^{*} \mathfrak{g}$ and denote the Hamiltonian vector field corresponding to $f$ by $X_{f}=\left(\zeta_{x}, \zeta_{p}\right)$.

We have:

$$
\begin{aligned}
d H . \delta \xi= & <f^{\prime}\left(\pi_{k}[p, x]\right), \delta \pi_{k}[p, x]> \\
= & <\pi_{k}\left(f^{\prime}\left(\pi_{k}[p, x]\right)\right),[\delta p, x]+[p, \delta x]> \\
& (\text { by the lemma) } \\
= & \omega\left(X_{f}, \delta \xi\right)=<\zeta_{x}, \delta p>-<\zeta_{p}, \delta x>.
\end{aligned}
$$

But $\pi_{k}\left(f^{\prime}\left(\pi_{k}[p, x]\right)\right)=f^{\prime}\left(\pi_{k}[p, x]\right)$. Hence the result.

Now consider the real Grassmannian $G_{q, n+1}(\mathbb{R})$ viewed as before as the symmetric space

$$
G_{q, n+1}(\mathbb{R})=S O(n+1) / S O(q) \times S O(p) \quad q+p=n+1, q \leq p .
$$

In this case we can write down the integrals quite explicitly. There are of course $p q$ integrals required. We take a somewhat different approach from Thimm.

As we did in Sect. 3 we represent elements of $G_{q, n+1}(\mathbb{R})$ by 2 by 2 block matrices in $s o(n+1)$ with zero diagonal blocks and nonzero off diagonal blocks, the upper right hand block being $p$ by $q$. We denote the matrices $p$ and $x$ in this case by $\hat{P}$ and $\hat{Q}$ as before. Then the geodesic flow is given as before by the double bracket equations (3.27). 
Now we consider the following sequence of projections: $\pi_{U}^{k}, 0 \leq k \leq p$ and $\pi_{L}^{k}, 0 \leq$ $k \leq q$ are the projections which eliminate the first $\mathrm{k}$ rows and columns of a matrix, and the rows and columns $q+1, \cdots, q+k$ of a matrix, respectively. In particular $\pi_{U}^{p}$ eliminates the upper $p$ by $p$ block and $\pi_{L}^{q}$ the lower $q$ by $q$ block. For example

$$
\pi_{U}^{1}\left[\begin{array}{llll}
a_{11} & a_{12} & \cdots & a_{1 n} \\
a_{21} & a_{22} & \cdots & a_{2 n} \\
\vdots & \vdots & \vdots & \vdots \\
a_{n 1} & a_{n 2} & \cdots & a_{n n}
\end{array}\right]=\left[\begin{array}{llll}
0 & 0 & \cdots & 0 \\
0 & a_{22} & \cdots & a_{2 n} \\
\vdots & \vdots & \vdots & \vdots \\
0 & a_{n 2} & \cdots & a_{n n}
\end{array}\right]
$$

We shall use this sequence of projections below to construct a chain of the type (5.44).

We begin by analyzing commutativity of flows on the Lie algebra. We then have

Theorem 5.3. The Hamiltonians

$$
\frac{1}{2 k} \operatorname{Tr}\left(\pi_{U}^{k} \pi_{L}^{j}[\hat{P}, \hat{Q}]\right)^{2 k}
$$

are all in involution on $T^{*} \mathfrak{g}$, where $\mathfrak{g}=s u(n+1)$ or $\operatorname{so}(n+1)$.

Proof. As before, the Hamiltonian flow of the integrals (5.51) is

$$
\dot{\hat{Q}}=\left[\hat{Q},\left(\pi_{U}^{k} \pi_{L}^{j}[\hat{P}, \hat{Q}]\right)^{2 l-1}\right] \quad \dot{\hat{P}}=\left[\hat{P},\left(\pi_{U}^{k} \pi_{L}^{j}[\hat{P}, \hat{Q}]\right)^{2 l-1}\right] .
$$

Then the Poisson bracket of any two flows with Hamiltonians $f$ and $g$ is of the form

$$
\begin{aligned}
\{f, g\} & =\omega(f, g) \\
& =<\left[\hat{Q},\left(\pi_{U}^{k} \pi_{L}^{j}[\hat{P}, \hat{Q}]\right)^{2 l-1}\right],\left[\hat{P},\left(\pi_{U}^{a} \pi_{L}^{b}[\hat{P}, \hat{Q}]\right)^{2 m-1}\right]> \\
& -<\left[\hat{P},\left(\pi_{U}^{k} \pi_{L}^{j}[\hat{P}, \hat{Q}]\right)^{2 l-1}\right],\left[\hat{Q},\left(\pi_{U}^{a} \pi_{L}^{b}[\hat{P}, \hat{Q}]\right)^{2 m-1}\right]> \\
& =<\left(\pi_{U}^{k} \pi_{L}^{j}[\hat{P}, \hat{Q}]\right)^{2 l-1},\left[[\hat{P}, \hat{Q}],\left(\pi_{U}^{a} \pi_{L}^{b}[\hat{P}, \hat{Q}]\right)^{2 m-1}\right]>
\end{aligned}
$$

by the Jacobi identity as before (the pairing here is the trace). Now choose $j \geq b$. Note that the upper blocks are paired with upper blocks and lower with lower under the pairing. Then if $k \geq a$, by repeated application of the lemma of Sect. 3 we get zero. If $k<a$, note that the pairing of the lower $2 \times 2$ block is of the form $<B^{k},\left[B, B^{a}\right]>$, where the superscripts denote the projected matrices. We can rewrite this $<B^{a},\left[B^{k}, B\right]>$ which again vanishes by the lemma.

This proves involution on the cotangent bundle of the algebra. However since the cotangent bundles of the Grassmannians are reduced submanifolds of the algebra and the integrals are invariants we obtain immediately involution on the Grassmannians.

To prove independence we need to find $p q$ independent integrals.

One method of seeing we have a sufficient number of integrals is to follow the sequence of projections onto to the descending chain of algebras $s o(n+1) \supset \operatorname{so}(n) \cdots s o(2)$ as discussed at the beginning of this section. In our setting this is implemented by projecting from upper left to lower right of the matrix $[\hat{P}, \hat{Q}]$ by a combination of upper and lower projections.

We then make the following observations: 
Independent integrals will come from invariant polynomials on the Lie algebra of $s o(n+1)$, then from those on $s o(n)$ and so on. The number of independent invariant polynomials on $s o(k)$ is equal to the dimension of the center of the enveloping algebra of $s o(k)$. For $s o(k)$ the dimension of the center is the integer part of $k / 2$. Restricted to the symmetric space $G_{q, n+1}(\mathbb{R})$, this gives a maximum of $q$, the rank of the symmetric space, independent functions at any point. We obtain $q$ such independent functions until we project to $s o(2 q-1)$ and below, in which case we obtain a maximal number of independent functions for $s o(k)$.

This gives us a total of $q(p-q+1)+(q-1)+(q-1)+(q-2)+(q-2) \cdots+1+1=$ $q(p-q+1)+2(q-1)+2(q-2) \cdots+2=p q$ integrals.

Note also that we are entitled to apply this argument to the functions arising from the bracket $[p, x]$ since for $s o(n)$ the derived algebra is equal to the algebra itself.

This proves complete integrability.

Example: For $s o(10)$ the dimensions of the centers of the enveloping algebra and its projections are: 544332211 . For $G_{3,10}(\mathbb{R})$ the corresponding count of independent invariant polynomials is: 333332211 . This gives us 21 functions as required.

We may also argue as follows:

Consider the two by two block structure of the matrices and recall that $q \leq p$. We consider the projections $\pi_{U}^{k}$ until $k=q$, leaving us with a square $2 q$ by $2 q$ matrix. Taking the invariant polynomials at each stage we obtain $q$ independent first integrals. Continuing with the upper projections we now get $q-1$ first integrals and then $q-2$ and so on. But by introducing now the lower projections at each stage we can double this.

Thus again we get a total of $q(p-q+1)+2(q-1)+2(q-2) \cdots+1+1=p q$ integrals.

A similar argument works for the complex Grassmannian - one obtains $2 p q$ integrals since there are double the number of invariant polynomials. (In particular in the polynomials in (5.51) we can replace $2 k$ by $k$.) Observe that this projection method in our setting provides a somewhat more direct method for obtaining all the integrals than the permutations invoked by Thimm, that the integrals are quite explicit, and give rise to explicit equations in the double double bracket form. Of course their existence may be deduced from the muliplicity free argument mentioned above.

Example: Observe also that for the particular case of $S^{n}\left(\mathbb{R P}^{n}\right)$ discussed above we obtain the standard integrals for the geodesic flow as discussed in Thimm [1981], but quite directly:

In this case the integrals are

$$
-\frac{1}{4} \operatorname{Tr}\left(\pi_{j}[\hat{P}, \hat{Q}]\right)^{2}=\frac{1}{4} \sum_{k, l \geq n+2-j}\left(q_{k} p_{l}-q_{l} p_{k}\right)^{2}, j=2, \cdots, n+1,
$$

where $\pi_{j} s o(n+1)=s o(j)$ is implemented by projection from the upper left. This implements the moment map discussed in Thimm directly.

Putting the involution and independence computations together we obtain the theorem of Thimm:

Theorem 5.4. The geodesic flow on the real and complex Grassmannians with respect to the "normal" metric is completely integrable. 


\section{References}

Abraham, R. and Marsden J. E.: Foundations of Mechanics, 2nd edition, Addison Wesley (1978)

Arnold, V.: Mathematical Method of Classical Mechanics, Springer-Verlag

Bloch, A. M.: Steepest descent, linear programming and Hamiltonian flows, Contemp. Math. AMS 114, 77-88 (1990)

Bloch, A.M.,Brockett, R.W. and Ratiu, T.S.: Completely integrable gradient flows. Commun. Math. Phys. 147, 57-74 (1992)

Bloch, A.M. and Crouch, P.E.: Optimal control and geodesic flows, Systems and Control Letters 28, 65-72 (1996)

Bloch, A.M. and Crouch, P.E.: Reduction of Euler Lagrange problems for constrained variational problems and relation with optimal control problems. Proc. CDC, 33, 2584-2590 (1994)

Bloch, A.M., Flaschka, H. and Ratiu, T.S.: A convexity theorem for isospectral sets of Jacobi matrices in a compact Lie algebra. Duke Math. J. 61, 41-66 (1990)

Brockett, R.W.: Lie theory and control systems defined on spheres. SIAM J. Appl. Math. 23, 213-225 (1973)

Brockett, R.W.: Dynamical systems that sort lists and solve linear programming problems. Proc. IEEE 27, 799-803 and Linear Algebra and its Appl. 146, (1991), 79-91 (1988)

Brockett, R.W.: Differential geometry and the design of gradient algorithms. Proc. Symp. Pure Math., AMS 54, Part I, 69-92 (1993)

Brockett, R.W.: The double bracket equation as a solution of a variational problem. Fields Institute Comm., 3, 69-76 (1994)

Crouch, P.E. and Silva Leite, F.: Geometry and the dynamic interpolation problem. Proc. A.C.C. Boston, 1131-1136 (1991)

Deift, P. A., L.-C. Li, Nanda, T. and Tomei, C.: The Toda lattice on a generic orbit is integrable, Comm. Pure Appl. Math. 39, 183-232 (1986)

Ercolani, N. M., Flaschka, H. and Singer, S.: The Geometry of the Full Toda Lattice (1992)

Faybusovich, L.E.: Explicitly solvable optimal control problems Int. J. Control 48 No. 6, 235-250 (1988)

Goldstein, H.: Classical Mechanics, Addison-Wesley (1980)

Guillemin, V. and Sternberg, S.: On collective integrability according to the method of Thimm Ergod. Th. and Dynam. Systems 3, 219-230 (1983)

Guillemin, V. and Sternberg, S.: Multiplicity-free spaces J. Diff. Geom. 19, 31-56 (1984)

Helgason, S.: Differential geometry, Lie groups, and symmetric spaces, Academic Press. (1978)

Howe, R.: Some highly symmetric dynamical systems, preprint. (1984)

Lu, J.-H. and Weinstein, A.: Poisson Lie groups dressing transformations, and the Bruhat decomposition, $J$. Diff. Geom 31, 501-526 (1990)

Marsden, J. E. and Ratiu, T.: Introduction to Mechanics and Symmetry, Springer Verlag (1994)

Mischenko, A. S. and Fomenko, A. T.: Integrability of Euler's equations on semisimple Lie algebras, Sel. Math. Sov. 2, 207-292

Mischenko, A. S. and Fomenko, A. T.: A generalized Liouville method for the integration of Hamiltonian systems, Functional analysis and its applications 12, 113-131 (1978)

Moser, J. Various aspects of integrable Hamiltonian systems. In Proc. CIME Conference, Bressanone, Italy 1978, Prog. Math. 8, Birkhauser. (1980)

Paternain, G. P and Spatzier, R. J.: New examples of manifolds with completely integrable geodesic flows Advances in Mathematics 108 No. 2, 346-365 (1994)

Ratiu, T.: The motion of the free n-dimensional rigid body, Indiana U. Math. J., 29, 609-627 (1980)

Thimm, A.: Integrable Hamiltonian systems on homogeneous spaces Ergod. Th. and Dynam. Systems 1, 495-517 (1981)

Communicated by S.-T. Yau 